\title{
THE MODIFIED PEIERLS-NABARRO MODEL OF INTERFACIAL MISFIT DISLOCATION
}

\author{
Y. YAO and T. C. WANG $\dagger$ \\ LNM, Institute of Mechanics, Chinese Academy of Sciences, Beijing 100080, People's Republic of \\ China
}

(Received 24 November 1998; accepted 24 April 1999)

\begin{abstract}
The Peierls-Nabarro model of the interfacial misfit dislocation array is analytically extended to a family of dislocations of greater widths. By adjusting a parameter, the width of the misfit dislocations, the distribution of the shear stress, and the restoring force law can be systematically varied. The smaller the amplitude of the restoring force, the wider the misfit dislocations and the lower the interfacial energy. (C) 1999 Acta Metallurgica Inc. Published by Elsevier Science Ltd. All rights reserved.
\end{abstract}

Keywords: Interface; Dislocations theory

\section{INTRODUCTION}

Interfaces play crucial roles in determining the properties and performance of materials. They may be completely incoherent, coherent but strained or semi-coherent. The incoherent and semi-coherent cases are characterized by the presence of misfit dislocations (MDs). These dislocations at the interface are geometrically necessary defects which are part of the interfacial structure, and they reduce the misfit strain with respect to the unrelaxed fully coherent interface by locally decreasing the interfacial coherency [1-3].

The concept of misfit dislocations was introduced by Frank and Van der Merwe [4,5] in a FrenkelKontorva model for oriented monolayer overgrowths in 1949. Since then there have been numerous observations of misfit dislocations in epitaxial thin films as reviewed by Matthews [6], and some observations in metal-ceramic composites have also been reported [7]. The Peierls-Nabarro (PN) model $[8,9]$, although relatively simple, can bridge continuum theory and atomistic theory, and has the potential of providing a quantitative estimation for the atomistic properties of the dislocations. Van der Merwe $[10,11]$ has studied interfacial MDs by means of Fourier analysis procedure. The Fourier analysis procedure is mathematically complex, and cannot provide a clear physical representation similar to the traditional treatment $[8,9]$. In our former work [12], we took an alternative simpler method than Van der Merwe's, in which we followed the original Peierls-Nabarro idea of dealing with a similar problem. Under the sinusoidal assumption for the law of force, an exactly analytic solution

$\dagger$ To whom all correspondence should be addressed. when the components of the bicrystal have different elastic parameters was obtained [12]. However, as pointed out by Foreman et al. [13], due to the assumed sinusoidal law of force, the dislocation core in the bulk dislocation will be extremely narrow. So a flatter type of periodic function may be valid in a real crystal. This paper treats the law of force for a flatter type, and represents a family of edge type misfit dislocations. We will also focus on how the interfacial energy depends on the law of force.

\section{PEIERLS-NABARRO MODEL}

As illustrated in Fig. 1, we consider a bicrystal composed of two cubic crystals 1 (upper) and 2 (lower) joined at the $\{001\}$ interface, and a MD array is positioned at the interface. We make the conventional assumptions: (i) the crystals deform under applied forces like isotropic elastic continua with shear moduli $\mu_{1}$ and $\mu_{2}$ and Poisson's ratios $v_{1}$ and $v_{2}$, respectively, (ii) crystals 1 and 2 have lattice parameters $a_{1}$ and $a_{2}$, respectively (assuming $a_{1}>a_{2}$ ), (iii) in order to achieve an appreciable mathematical simplification of the problem, the lattices of materials 1 and 2 are imagined to be generated from a so-called reference lattice with lattice parameter $c$ defined by [10]

$$
p=P a_{1}=(P+1) a_{2}=\left(P+\frac{1}{2}\right) c
$$

where $P$ is an integer. Equation (1) thus defines the misfit $f, c$, and the MD spacing $p$ as

$$
p=\frac{a_{1} a_{2}}{a_{1}-a_{2}}, c=\frac{2 a_{1} a_{2}}{a_{1}+a_{2}}, f=\frac{c}{p}=\frac{2\left(a_{1}-a_{2}\right)}{a_{1}+a_{2}} .
$$




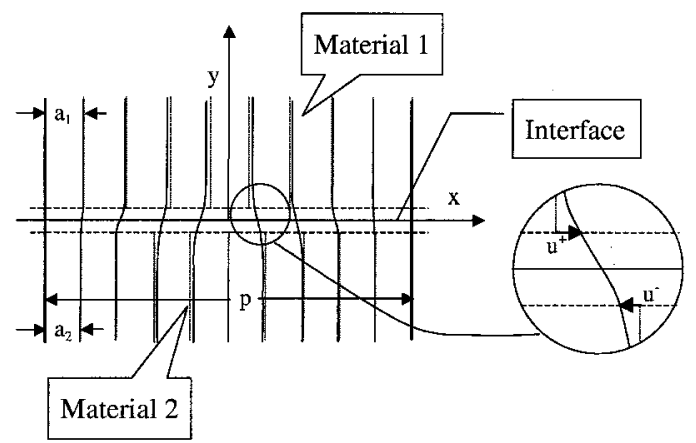

Fig. 1. The misfit edge dislocation at the interface.

The definition of the misfit is very close to a conventional definition $\left(a_{1}-a_{2}\right) / a_{2}$ in thin films when $a_{1}$ and $a_{2}$ are very close. As pointed out by Van der Merwe [14], it seems meaningful to use the quantity $c / p$ rather than $\left(a_{1}-a_{2}\right) / a_{2}$ as a measure of misfit when both crystals are semi-infinite.

As shown in Fig. 1, we assume the dislocation core to be confined within the glide plane $x z$ (i.e. interface), and the dislocation line to be chosen as the $z$-axis direction. When neglecting the normal component of the Burgers vector of dislocations at the interface, the Burgers vector $\vec{b}\left(=\vec{u}^{+}-\vec{u}^{-}\right)$is only parallel to the $x$-axis. For a single edge dislocation at the interface, within a continuum theory, the relevant shear stress at the interface is

$$
\sigma_{x y}=\frac{2 \mu_{2}}{\left(\kappa_{2}+1\right)} \frac{\left(1+\alpha_{1}\right)}{\left(1-\alpha_{2}^{2}\right) \pi}\left(\frac{b}{x}\right)
$$

where $\alpha_{1}$ and $\alpha_{2}$ are Dundurs' parameters. $\dagger$ For the periodic dislocation array in Fig. 1, the relevant shear stress along the interface is

$$
\sigma_{x y}=\frac{2 \mu_{2}}{\left(\kappa_{2}+1\right)} \frac{\left(1+\alpha_{1}\right) b}{\left(1-\alpha_{2}^{2}\right) p} \cot \left(\frac{\pi x}{p}\right) .
$$

Following the original PN model [8,9], a continuous distribution of infinitesimal dislocations with Burgers vector density $\rho\left(x^{\prime}\right)=-\mathrm{d} u\left(x^{\prime}\right) / \mathrm{d} x^{\prime}$ can produce the plastic displacement $u(x)\left[=u^{+}(x)-u^{-}(x)\right]$ of the upper part of the interface $(y>0)$ with respect to the lower part $(y<0)$. The resultant shear stress at $x, \sigma_{x y}(x)$, is the sum of the contributions from all these infinitesimal dislocations. $\sigma_{x y}(x)$ is balanced by the corresponding component of the periodic restoring force $F(U(x))$ acting between atoms on either side of the interface. $U(x)$, composed of plastic displacement and misfit displacement due to $a_{1} \neq a_{2}$, is the relative displacement of an atom in material 1 relative to the corresponding atom in material 2

$\dagger \alpha_{1}=\left[\Gamma\left(\kappa_{2}+1\right)-\left(\kappa_{1}+1\right)\right] /\left[\Gamma\left(\kappa_{2}+1\right)+\left(\kappa_{1}+1\right)\right]$, $\alpha_{2}=\left[\Gamma\left(\kappa_{2}-1\right)-\left(\kappa_{1}-1\right)\right] /\left[\Gamma\left(\kappa_{2}+1\right)+\left(\kappa_{1}+1\right)\right], \quad$ and $\Gamma=\mu_{1} / \mu_{2}, \kappa_{1}=3-4 v_{1}, \kappa_{2}=3-4 v_{2}$ for plane strain For the derivation of equation (3) refer to Ref. [15].

$$
U(x)=\frac{c}{2}+\frac{c}{p} x+u(x)
$$

The term $c / 2+c x / p$ simply represents the relative displacement of the corresponding atoms when the bicrystal is constructed in an imagined generation process without any deformation. For the case of a periodic MD array at the interface, using equation (4) we can obtain the following integrodifferential equation similar to the PN equation:

$$
-\mu \int_{-p / 2}^{p / 2} \frac{1}{p} \cot \left[\frac{\pi\left(x-x^{\prime}\right)}{p}\right] \frac{\mathrm{d} u\left(x^{\prime}\right)}{\mathrm{d} x^{\prime}} \mathrm{d} x^{\prime}=F(U(x))
$$

with the boundary conditions $u(-p / 2)=0$ and $u(p / 2)=0$. The value of $\mu$ depends on the elastic constants of two materials, and is equal to $2 \mu_{2}\left(1+\alpha_{1}\right) /\left[\left(\kappa_{2}+1\right)\left(1-\alpha_{2}^{2}\right)\right]$. Similar to the original PN model, the restoring stress $F(U)$ may assume a sinusoidal form

$$
F(U(x))=\frac{\tau_{0}}{2 \pi} \sin [2 \pi U(x) / c]
$$

and $\tau_{0}$ is defined as the shear modulus parallel to the interface.

With the help of equations (5) and (7), equation (6) can be transformed into

$$
\begin{aligned}
& -\mu \int_{-p / 2}^{p / 2} \frac{1}{p} \cot \left[\frac{\pi\left(x-x^{\prime}\right)}{p}\right] \frac{\mathrm{d} U\left(x^{\prime}\right)}{\mathrm{d} x^{\prime}} \mathrm{d} x^{\prime} \\
& =\frac{\tau_{0}}{2 \pi} \sin [2 \pi U(x) / c]
\end{aligned}
$$

where boundary conditions are $U(-p / 2)=0$ and $U(p / 2)=c$. In general, there is no systematic method to solve the integro-differential equation (8). However, in Ref. [12], we had guessed that the integro-differential equation (8) had the following analytic solution:

$$
U(x)=\frac{c}{2}+\frac{c}{\pi} \arctan \left[\left(\sqrt{\beta^{-2}+1}+\beta^{-1}\right) \tan \frac{\pi x}{p}\right]
$$

where

$$
\beta=\frac{2 \pi c \mu}{p \tau_{0}}=\frac{2 \pi \mu f}{\tau_{0}} .
$$

Here, the dimensionless number $\beta$ is a key physical parameter which controls the structure of the interfaces. $\beta$ decreases with increasing $\tau_{0}$ and decreasing $f$. From equation (5), we can obtain the plastic displacement

$$
\begin{aligned}
u(x)= & \frac{c}{\pi} \arctan \left[\left(\sqrt{\beta^{-2}+1}+\beta^{-1}\right) \tan \frac{\pi x}{p}\right] \\
& -\frac{c}{p} x .
\end{aligned}
$$

It is worth noting that the solution of equation (9) is completely similar to the solution of Van der Merwe [10,11]. But the governing equation of Van der Merwe's solution takes the form $[10,11]$ 


$$
\begin{aligned}
2 \pi\left[\frac{\mathrm{d} \psi}{\mathrm{d} X}-\frac{1}{2 \pi}\right]= & -\frac{1}{2 \pi \beta} \int_{0}^{\pi}[\sin 2 \pi \psi(X+t) \\
& -\sin 2 \pi \psi(X-t)] \cot \frac{1}{2} t \mathrm{~d} t
\end{aligned}
$$

where $\psi=U / c, X=2 \pi x / p$.

Equation (12) completely differs from our governing equation (8). The governing equation (12) was solved by Van der Merwe through Fourier analysis. Van der Merwe's result is an approximate solution, in which normal displacement is not continuous across the interface. In our derivation, the continuity of tractions and displacements at the interface is assured, since equation (3) is the exact elastic stress solution of an edge dislocation at the interface. The difference between our solution and Van der Merwe's solution is that $\mu=1 /\left[\left(1-v_{1}\right) / \mu_{1}+(1-\right.$ $\left.v_{2}\right) / \mu_{2}$ ] in Van der Merwe's method, while in our method $\quad \mu=2 \mu_{2}\left(1+\alpha_{1}\right) /\left[\left(\kappa_{2}+1\right)\left(1-\alpha_{2}^{2}\right)\right]$. Apparently they are equal only when the two components of the interface have the same elastic parameters; otherwise, they are not equal, and the relative difference between them may be up to $13 \%$.

Similar to bulk dislocation, the half-width of the dislocation $\zeta$ can be defined as

$$
\zeta=\frac{2 c}{\pi f} \arctan \left[\left(\sqrt{\beta^{2}+1}-1\right) / \beta\right] .
$$

Clearly, the dislocation core width is related to the misfit $f$, shear modulus $\tau_{0}$ and elastic constants of two materials. This illustrates the fact that misfit $f$ and shear modulus $\tau_{0}$ can influence the interface structure. Equation (13) shows that an increase in shear modulus $\tau_{0}$ leads to a decrease in width of the misfit dislocation core, whereas at constant shear modulus $\tau_{0}$, the width of a dislocation increases with decreasing of misfit $f$. When $\tau_{0}=2 \mu$ and $f=0.05, \zeta \doteq c$, i.e. about 1.0 atomic spacings, it is very narrow. The misfit dislocations are wider than this in an actual bicrystal. The extreme narrowness here is due to the assumed sinusoidal law of force equation (7). Therefore, to consider a flatter type of periodic function of stress against relative displacement may be more valuable.

\section{MODIFICATION OF THE LAW OF FORCE}

As mentioned above, the assumed sinusoidal law of the restoring force equation (7) is unlikely to be valid in real crystals. As a consequence, a flatter type of periodic function of stress against relative displacement should be proposed, which will lead to a wider dislocation core width. In the following, we will investigate this question quantitatively, and determine how the width of a dislocation and the interfacial energy vary with the assumed law of forces.

In order to obviate the difficulty of having to solve the integral equation (6) of the type mentioned, similar to Foreman et al.'s treatment [13], the method of approach adopted here is to choose some suitable type of function $U(x)$, to find the corresponding stress distribution from equation (6) and, by eliminating $x$ between them, find $F(U)$ as a function of $U$. The main difficulty of the approach is to find some type of function $U(x)$ which will ensure that the initial slope of the corresponding function is a constant $\tau_{0}$ for a small value of $U / c$ at the origin $x=0$.

As has been stated, it is required to find such displacement functions $U(x)$ that they (i) represent wider dislocations than that obtained by assumed sinusoidal force, and (ii) give curves of stress against relative displacement having the same initial slope as the previously assumed sinusoidal curve equation (7), thus ensuring that $F(U)$ is the same for small $U(x) / c$.

A displacement function which satisfies the conditions mentioned is

$$
\begin{aligned}
U(x)= & \frac{c}{2}+\frac{c}{\pi} \arctan \left[\frac{1}{a} \tan \frac{\pi x}{p}\right] \\
& -f(a) \frac{a^{-2} \tan \frac{\pi x}{p}}{1+a^{-2} \tan ^{2}\left(\frac{\pi x}{p}\right)}
\end{aligned}
$$

where $f(a)=\left(\beta-2 a-\beta a^{2}\right) /(2 a \beta+2)$. When $\beta-2 a-\beta a^{2}=0$, namely $a=a_{0}=1 /\left(\sqrt{\left(\beta^{-2}+1\right)}+\right.$ $\left.\beta^{-1}\right), U(x)$ reduces to equation (9). Displacement curves $U(x)$ with various values of $a$ are shown in Fig. 2 for the cases $\beta=0.1$ and 1.0. Clearly, the larger $a$ is, the wider the dislocation is. In the wider dislocations, $U(x)$ varies more slowly from atom to atom, so that the maximum elastic strains are smaller, and the theory may be accordingly more consistent. Equation (14) can be conveniently written as

$$
U(x)=\frac{c}{2}+\left\{1+f(a) \frac{\partial}{\partial a}\right\} \frac{c}{\pi} \arctan \left[\frac{1}{a} \tan \frac{\pi x}{p}\right] .
$$

Inserting equation (15) into the left-hand side of equation (8), the resulting stress distribution is then

$$
\sigma_{x y}=-\frac{c \mu}{p}\left[\frac{\left(a^{-2}-1\right) \tan \left(\frac{\pi x}{p}\right)}{1+a^{-2} \tan ^{2}\left(\frac{\pi x}{p}\right)}-f(a) \frac{2 a^{-3} \tan \left(\frac{\pi x}{p}\right)+2 a^{-3} \tan ^{3}\left(\frac{\pi x}{p}\right)}{\left[1+a^{-2} \tan ^{2}\left(\frac{\pi x}{p}\right)\right]^{2}}\right] .
$$




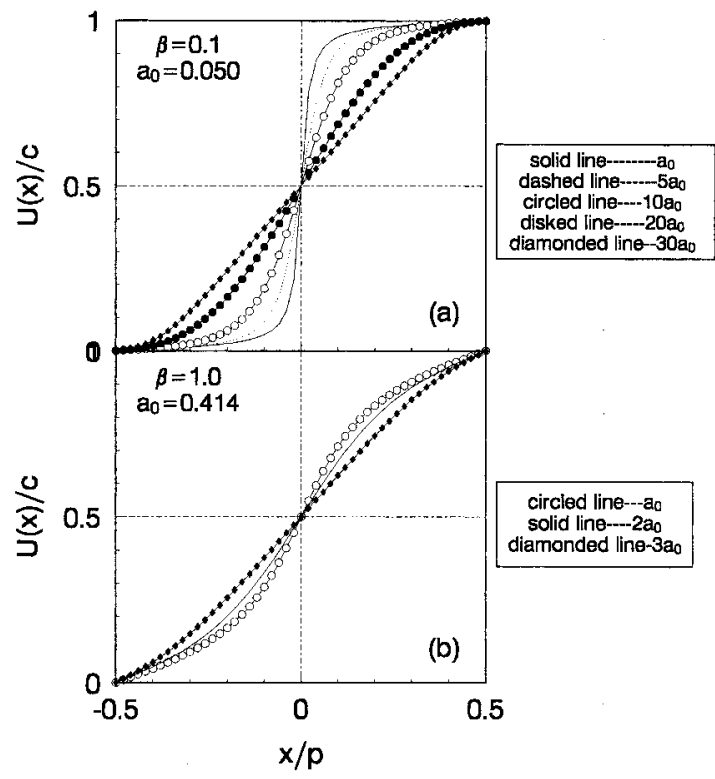

Fig. 2. The displacement curves for various values of $\beta$ and $a . U(x)$ is the atomic relative displacement function between corresponding atoms at the interface, $x$ the atom coordinate, $c$ the lattice parameter of the reference lattice, and $p$ the MD spacing. So both $U / c$ and $x / p$ are dimensionless quantities. Upper figure: $\beta=0.1$ and $a=a_{0}, 5 a_{0}$, $10 a_{0}, 20 a_{0}, 30 a_{0}\left(a_{0}=0.050\right)$. Lower figure: $\beta=1.0$ and $a=a_{0}, 2 a_{0}, 3 a_{0}\left(a_{0}=0.414\right)$

The shear stress curves are shown in Fig. 3 for the cases $\beta=0.1$ and 1.0. As the dislocation widens, along with increasing $a$, it is found that maximum stress decreases, its position moves outwards and the shape of the curve is smoother.

Considering equations (14) and (16), the function $F(U)$ corresponding to the assumed displacement is written most conveniently in parametric form as

$$
\begin{gathered}
U(\theta)=\theta+\frac{f(a)}{2 a} \frac{c}{\pi} \sin \left(\frac{2 \pi}{c} \theta\right) \\
F(\theta)=-\frac{c \mu}{p}\left[\left(\frac{a}{2}-\frac{1}{2 a}\right) \sin \frac{2 \pi}{c} \theta+\frac{f(a)}{2} \sin \frac{4 \pi}{c} \theta\right. \\
\left.+f(a)\left(a^{-2}+1\right) \sin \frac{2 \pi}{c} \theta^{*} \sin ^{2}\left(\frac{\pi}{c} \theta\right)\right]
\end{gathered}
$$

where

$$
\theta=\frac{c}{2}+\frac{c}{\pi} \arctan \left[\left(\tan \frac{\pi x}{p}\right) / a\right] .
$$

The cases $\beta=0.1$ and 1.0 are illustrated in Fig. 4. As can be seen, the initial slopes of $F(U)$ curves are constant $\tau_{0}=(2 \pi \mu f) / \beta$ for all values of $a$ when $\beta$ is fixed, but amplitudes decrease as $a$ increases, i.e. the misfit dislocation width increases. This can be shown clearly in Tables 1 and 2. For example, when $\beta=0.1, a=5 a_{0}\left(a_{0}=0.050\right)$, the amplitude of the restoring force becomes 0.39 times that of sinusoidal law, then the width of dislocation is 2.5 times that of sinusoidal law. The width of misfit dis-

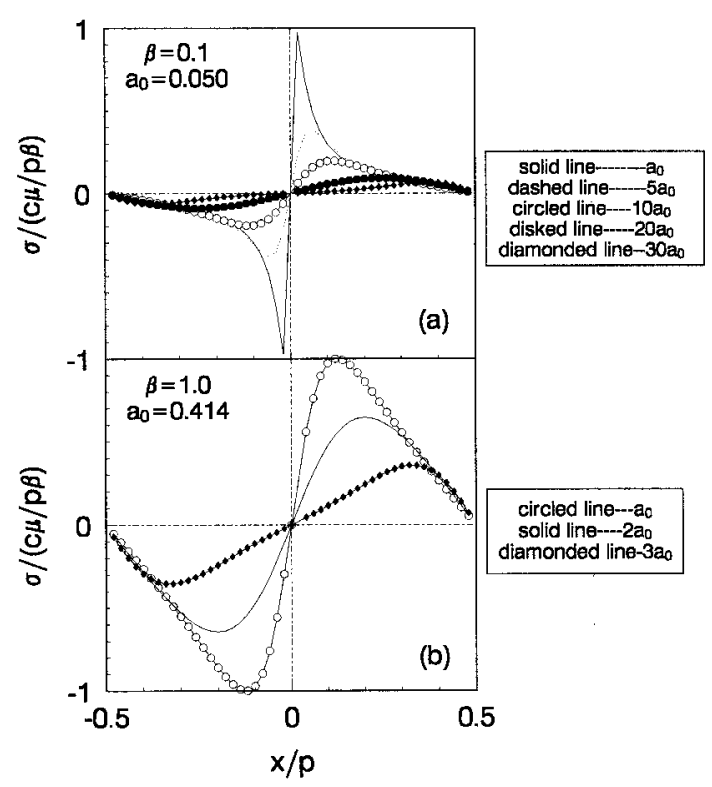

Fig. 3. The corresponding shear stress $\sigma$ distributions with respect to displacements in Fig. 2 for various values of $\beta$ and $a . \sigma /(c \mu / p \beta)$ is a dimensionless quantity. All the other parameters are the same as in Fig. 2.

locations is approximately linear inversely proportional to the amplitude of the restoring force when $a$ is between $a_{0}$ and $10 a_{0}$ for $\beta=0.1$, i.e. $\zeta^{*} F_{\max } \approx c \mu / \pi$, and this result was also found in the bulk dislocation by Foreman et al. [13]. However, this linear inversely proportional relation seems not satisfactory when $\beta$ is large, for example, when $\beta=1.0, a=3 a_{0}\left(a_{0}=0.414\right)$, the amplitude of the restoring force becomes 0.36 times that of sinusoidal law, but the width of dislocation is 1.64 times that of sinusoidal law.

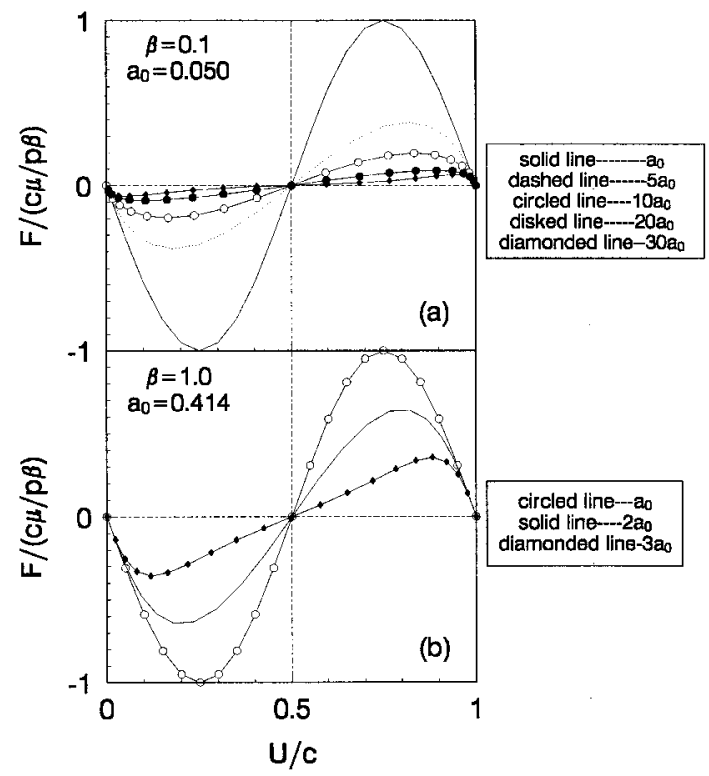

Fig. 4. Curves of stress $F$ against displacement $U$. $F /(c \mu / p \beta)$ is a dimensionless quantity. All the other parameters are the same as in Fig. 2. 
Table 1. Information of misfit dislocations for $\beta=0.1$. Here, $a_{0}=0.050 ; \zeta$ is the half-width of misfit dislocations; $F_{\max }$ the maximum of restoring stress $F(U) ; E_{\mathrm{m}}, E_{\mathrm{e}}, E$ the misfit energy, the elastic energy and the total interfacial energy, respectively. The definitions of $c, \mu, p$ and $\beta$ can be found in the text

\begin{tabular}{|c|c|c|c|c|c|}
\hline$a$ & $\zeta / p$ & $F_{\max } /(c \mu / p \beta)$ & $E_{\mathrm{m}} /\left(c^{2} \mu / 2 \pi p \beta\right)$ & $E_{\mathrm{e}} /\left(c^{2} \mu / 2 \pi p \beta\right)$ & $E /\left(c^{2} \mu / 2 \pi p \beta\right)$ \\
\hline$a_{0}$ & 0.032 & 1.0 & 0.095 & 0.17 & 0.27 \\
\hline $5 a_{0}$ & 0.080 & 0.39 & 0.093 & 0.11 & 0.20 \\
\hline $10 a_{0}$ & 0.15 & 0.20 & 0.087 & 0.060 & 0.15 \\
\hline $20 a_{0}$ & 0.28 & 0.091 & 0.070 & 0.020 & 0.090 \\
\hline $30 a_{0}$ & 0.39 & 0.035 & 0.051 & 0.0065 & 0.058 \\
\hline
\end{tabular}

Table 2. Information of misfit dislocations for $\beta=1.0$. Here, $a_{0}=0.414$ and the definitions of the rest of the physical quantities are the same as in Table 1

\begin{tabular}{|c|c|c|c|c|c|}
\hline$a$ & $\zeta / p$ & $F_{\max } /(c \mu / p \beta)$ & $E_{\mathrm{m}} /\left(c^{2} \mu / 2 \pi p \beta\right)$ & $E_{\mathrm{e}} /\left(c^{2} \mu / 2 \pi p \beta\right)$ & $E /\left(c^{2} \mu / 2 \pi p \beta\right)$ \\
\hline$a_{0}$ & 0.25 & 1.0 & 0.59 & 0.19 & 0.78 \\
\hline $2 a_{0}$ & 0.32 & 0.65 & 0.50 & 0.10 & 0.60 \\
\hline $3 a_{0}$ & 0.41 & 0.36 & 0.33 & 0.027 & 0.36 \\
\hline
\end{tabular}

\section{DISCUSSION AND APPLICATION}

\subsection{Energy of misfit dislocation}

Similar to the original PN model, the energy of the misfit dislocation is calculated as the sum of two contributions: the elastic strain energy stored in the two half-crystals and the misfit energy associated with the nonlinear distortion of bonds across the slip plane [16]. With the help of equations (14) and (16), the elastic strain energy per unit area can be obtained from

$$
E_{\mathrm{e}}=-\frac{1}{2 p} \int_{-p / 2}^{+p / 2} \sigma_{x y}(x) u(x) \mathrm{d} x .
$$

The misfit energy per unit area can be written as

$$
E_{\mathrm{m}}=\frac{1}{p} \int_{-p / 2}^{+p / 2} \Phi[U(x)] \mathrm{d} x .
$$

In parametric form, the misfit energy per unit area can be expressed as

$$
E_{\mathrm{m}}=\frac{1}{p} \int_{-p / 2}^{+p / 2} \Phi[\theta(x)] \mathrm{d} x
$$

where

$$
\Phi(\theta)=-\int F(\theta) \mathrm{d} U(\theta)
$$

Therefore, the mean total energy per unit area of the interface due to forming of misfit dislocation is called interfacial energy and it can be written as

$$
E=E_{\mathrm{e}}+E_{\mathrm{m}} .
$$

Only if $F(U)$ is adopted as sinusoidal law of force, the interfacial energy can be integrated as

$$
E=\frac{\tau_{0} c}{4 \pi^{2}}\left(1+\beta-\sqrt{1+\beta^{2}}-\beta \ln \left[2 \beta \sqrt{1+\beta^{2}}-2 \beta^{2}\right]\right) .
$$

Usually, the interfacial energy $E$ has to be numerically calculated, the resulting interfacial energy $E$ is shown in Figs 5 and 6 for various $\beta$ and $a$. As $a$ increases, the elastic energy, the misfit energy and the total energy decrease when $\beta$ is fixed. The elastic energy decreases more rapidly than the misfit energy as $a$ increases for fixed $\beta$. This means that

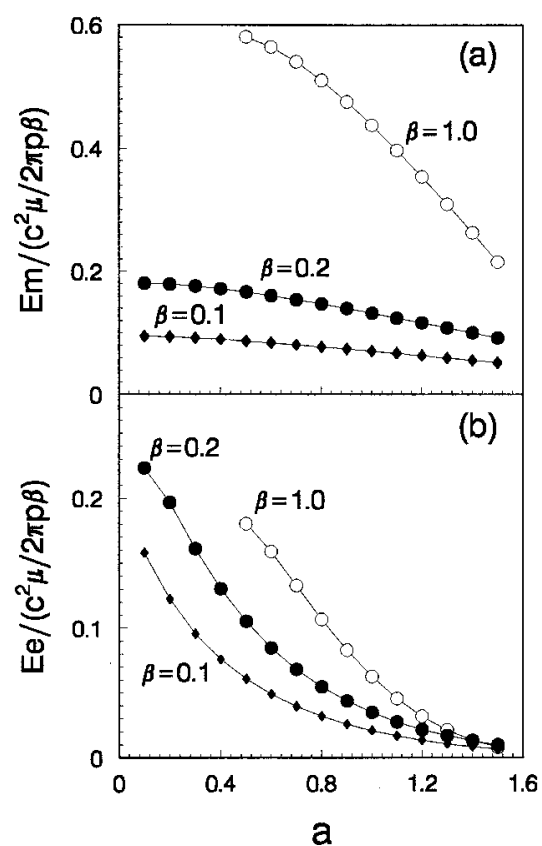

Fig. 5. Upper diagram: the misfit energy $E_{\mathrm{m}}$ vs $a$ for $\beta=0.1,0.2$, and 1.0. Lower diagram: the elastic energy $E_{\mathrm{e}}$ vs $a$ for $\beta=0.1,0.2$, and 1.0 . Both $E_{\mathrm{e}} /\left(c^{2} \mu / 2 \pi p \beta\right)$ and $E_{\mathrm{m}} /\left(c^{2} \mu / 2 \pi p \beta\right)$ are dimensionless quantities. 


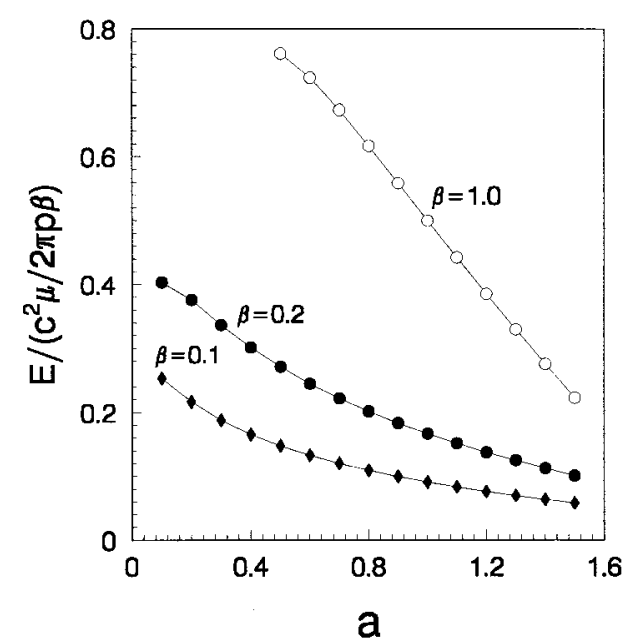

Fig. 6. The interfacial energy $E$ vs $a$ for $\beta=0.1,0.2$, and 1.0. $E /\left(c^{2} \mu / 2 \pi p \beta\right)$ is a dimensionless quantity.

interfacial energy depends on both the magnitude of restoring force $F$ and the form of force. These can be shown clearly in Tables 1 and 2. For example, when $\beta=0.1, a=5 a_{0} \quad\left(a_{0}=0.050\right)$, the width of dislocation increases 1.5 times that of sinusoidal law, here the misfit energy only decreases by $2 \%$ compared with that of sinusoidal law, but the elastic energy decreases by $35 \%$. The misfit energy, elastic energy and interfacial energy decrease more rapidly for larger $\beta$ than smaller $\beta$. For example, when $\beta=1.0, a=3 a_{0} \quad\left(a_{0}=0.414\right)$, the width of dislocation increases 0.64 times that of sinusoidal law, here the misfit energy decreases by $44 \%$ compared with that of sinusoidal law, but the elastic energy decreases by $86 \%$. This shows that energies are more dependent on the form of restoring force for larger $\beta$ than for smaller $\beta$.

\section{SUMMARY AND CONCLUSIONS}

The Peierls-Nabarro model for the interfacial misfit dislocation array is analytically extended to a family of dislocations of greater widths. We can systematically vary the width of misfit dislocations, the distribution of shear stress, and the law of restoring force by adjusting parameter $a$. As $a$ increases, the amplitudes of the restoring force and shear stress of the misfit dislocation decreases and the width of dislocation increases. The width of misfit dislocations is approximately linear inversely proportional to the amplitude of the restoring force when $a$ is between $a_{0}$ and $10 a_{0}$ for smaller $\beta$, i.e. $\zeta^{*} F_{\max } \approx c \mu / \pi$. Meantime, the elastic energy, the misfit energy and the total energy decrease as $a$ increases when $\beta$ is fixed. The energies depend on the form of the restoring force, and the dependence is stronger for the case of large $\beta$ than that of small $\beta$. In further work, we will discuss the solution of equation (6) for the arbitrary restoring force $F(U)$.

Acknowledgements-The research presented here was supported by the National Natural Science Foundation of China (No. 19704100) and National Science Foundation of Chinese Academy of Sciences (project KJ951-1-201).

\section{REFERENCES}

1. Hirth, J. P. and Feng, X., J. appl. Phys., 1992, 67, 3343.

2. Feng, X. and Hirth, J. P., J. appl. Phys., 1992, 72, 1386.

3. Kirchner, H. O. K., Physica Scripta, 1992, 44, 151.

4. Frank, F. C. and Van der Merwe, J. H., Proc. R. Soc. Lond. Ser. A, 1949, 198, 205.

5. Frank, F. C. and Van der Merwe, J. H., Proc. R. Soc. Lond. Ser. A, 1949, 198, 216.

6. Matthews, J. W., in Dislocations in Solids, Vol. 2, ed. F. R. N. Nabarro. North-Holland, Amsterdam, 1979, p. 461.

7. Vitek, V., Gutekunst, G., Mayer, J. and Rühle, M., Phil. Mag. A, 1995, 71, 1219.

8. Peierls, R., Proc. Phys. Soc. Lond., 1940, 52, 34.

9. Nabarro, F. R. N., Proc. Phys. Soc. Lond., 1947, 59, 256.

10. Van der Merwe, J. H., Proc. Phys. Soc. Lond. A, $1950,63,616$.

11. Van der Merwe, J. H., J. appl. Phys., 1963, 34, 117.

12. Yao, Y., Wang, T. C. and Wang, C. Y., Phys. Rev. B, $1999, \mathbf{5 9}, 8232$.

13. Foreman, A. J., Jaswon, M. A. and Wood, J. K., Proc. R. Soc. A, 1951, 64, 156.

14. Van der Merwe, J. H., in Treatise on Materials Science and Technology, Vol. 2, ed. H. Herman. Academic Press, New York, 1973, p. 1.

15. Suo, Z. and Hutchinson, J. W., Int. J. Fract., 1990, $\mathbf{4 3}, 1$.

16. Hirth, J. P. and Lothe, J., Theory of Dislocations, 2nd edn. Wiley, New York, 1982, pp. 226-237. 